P150 PERCEPTIONS OF ASTHMA CONTROL IN THE UK - A CROSS SECTIONAL STUDY COMPARING PATIENT AND HCP PERCEPTIONS OF ASTHMA CONTROL WITH VALIDATED ACT SCORES

${ }^{1} \mathrm{~A}$ Menzies-Gow, ${ }^{2} \mathrm{G}$ Chiu. ${ }^{1}$ Royal Brompton Hospital, London, UK; ${ }^{2}$ Boehringer Ingelheim UK, Bracknell, UK

10.1136/thoraxjnl-2016-209333.293

Uncontrolled asthma is a major health problem. Personal perceptions of asthma control often vary between patients and their treating physicians, and both may differ from actual control. This can be a major barrier in optimising patient asthma care.

The aim of this cross-sectional survey was to provide UK-specific data on actual and perceived asthma control in a sample of adult (18-75 years) asthma patients attending routine asthma reviews in primary, secondary and tertiary settings. Differences between healthcare professionals' (HCP) and patients' perceived assessments of asthma control were evaluated via an online questionnaire and compared to a control - the validated Asthma Control Test (ACT) questionnaire, which was completed by the patient.

Patients with a documented diagnosis of asthma who were taking medication (at least a short acting ß-agonist) were enrolled and consented by their HCP within a month of their last clinic appointment. Individuals with a history of an asthma exacerbation within prior 4 weeks; a diagnosis of another respiratory condition; or a smoking history $>10$ pack years were excluded. Patients were grouped into BTS/SIGN treatment Steps 1-5.

260 patients were screened. 234 patients were eligible for the study: 33, 52, 50, 49 and 50 patients in Steps 1 to 5 , respectively. Women composed $70 \%$ (164) of the study population. $47.4 \%$ of patients were aged 45-64 years. 164 patients (70\%) were classed as non-smokers by HCPs.

The ACT results suggest that asthma was only controlled in $54.7 \%$ of patients overall (defined as ACT score $\geq 20$ ), with levels of uncontrolled asthma highest in Step 4-5 patients. This is in contrast to $84.2 \%$ of patients and $73.9 \%$ of HCPs who perceived that asthma was controlled.

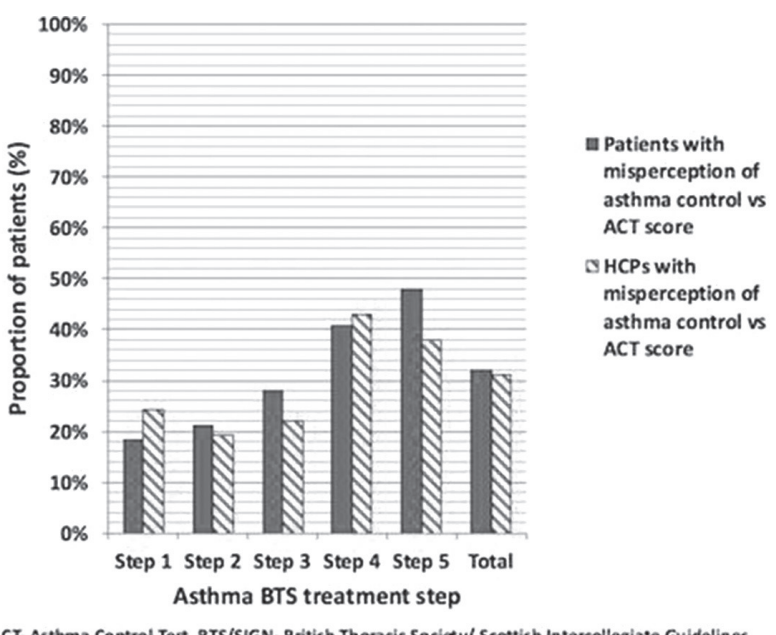

ACT- Asthma Control Test, BTS/SIGN-British Thoracic Society/ Scottish Intercollegiate Guidelines Network, HCP. Healthcare professional, percentages are based on the number of patients in the full analysis set. BTS Step $1=$ Mild intermittent asthma, Step $2=$ Regular preventer therapy, Step $3=$ Initial add-on therapy, Step 4= Persistent poor control, Step $5=$ continuous or frequent use of oral steroids

Abstract P150 Figure 1 Perception of asthma control did not correlate with ACT score: Patients vs HCPs

These data suggest a high level of uncontrolled asthma in UK asthma patients, especially Step 4-5 patients. A significant proportion of both patients and HCPs may have an incorrect perception of asthma control, representing a significant unmet medical need in terms of optimal asthma management.

Overall, correct correlation of ACT score with perception of controlled or uncontrolled asthma only occurred in $67.9 \%$ of patients and $68.8 \%$ of HCPs. The poorest correlations occurred in Step 4-5 patients.

\section{P151 WITHDRAWN: EFFECT OF HIGH-INTENSITY EXERCISE ON LUNG FUNCTION, AEROBIC PERFORMANCE AND AIRWAY INFLAMMATION IN ASTHMA}

${ }^{1} \mathrm{C}$ Winn, ${ }^{1} \mathrm{M}$ McNarry, ${ }^{1} \mathrm{G}$ Stratton, ${ }^{2} \mathrm{AM}$ Wilson, ${ }^{1} \mathrm{GA}$ Davies. ${ }^{1}$ Swansea University, Swansea, UK; ${ }^{2}$ University of East Anglia, Norwich, UK

10.1136/thoraxint-2016-209333.294

\section{P152 INHALED CORTICOSTEROID (ICS) AND LONG ACTING BETA-ADRENOCEPTOR AGONIST (LABA) THERAPY ADHERENCE REPORTING AND MONITORING IN CLINICAL TRIALS OF SEVERE ADULT ASTHMA DRUG TREATMENTS: A SYSTEMATIC REVIEW}

${ }^{1} \mathrm{MC}$ Mokoka, ${ }^{2} \mathrm{MJ}$ MCDonnell, ${ }^{1} \mathrm{~B}$ Cushen, ${ }^{1} \mathrm{~S}$ Cormican, ${ }^{1}$ I Sulaiman, ${ }^{1} \mathrm{~F}$ Doyle, ${ }^{1} \mathrm{~F}$ Boland, ${ }^{1}$ RW Costello. ${ }^{1}$ Royal College of Surgeons of Ireland, Dublin, Ireland; ${ }^{2}$ National University of Ireland, Galway, Ireland

\subsection{6/thoraxjnl-2016-209333.295}

Background Assessment of adherence to inhaled corticosteroids and long-acting beta-agonist therapy, allows identification of patients classified as having refractory asthma. It is crucial to ensure that adherence is adequately assessed in clinical practice and in the conduct of clinical trials to target the patients who may benefit from expensive potential add-on therapies. We hypothesised that adherence to inhaled corticosteroids and long acting $\beta_{2}$-agonists is under-assessed and under-reported in clinical trials of add-on drug treatment interventions in adolescent and adult patients with severe asthma.

Methods A systematic literature search of six major databases was performed to identify randomised controlled trials (RCTs) of asthma drug treatment interventions conducted in severe adolescent and adult asthma patients taking inhaled corticosteroids (ICS) alone or in combination with long-acting beta-agonist therapy (ICS/LABA). Identified studies were reviewed concerning key characteristics of the trial and the intervention; reporting and monitoring of adherence to ICS/LABA and the relationship between measuring adherence and study outcomes was assessed. This systematic review had been registered on PROSPERO; registration number CRD42015029611.

Results The electronic search retrieved 5869 articles with an additional 19 identified from references. 4008 articles were screened after removal of duplicates of which 72 RCTs were included and underwent data extraction and quality scoring. Of these, only 12 RCTs reported adherence to ICS or ICS/LABA therapy. Measures of adherence used included; self-report, $\mathrm{n}=1$; self-report and inhaler technique, $\mathrm{n}=1$; inhaler technique, $\mathrm{n}=1$; inhaler technique and FeNO, $\mathrm{n}=1$; dose counting. $\mathrm{n}=1$; diary, $\mathrm{n}=2$; prescription records, $\mathrm{n}=1$; weighing inhaler canister, $\mathrm{n}=1$; assumption that primary respiratory physician had assessed adherence, $n=1$; and method of adherence assessment not reported but measure of adherence included in $\mathrm{n}=2$ studies. High levels of heterogeneity across studies with 
regard to adherence and exacerbation measurements, designs and analysis precluded a formal meta-analysis. Although effect measures varied, good adherence was associated with fewer severe asthma exacerbations in high-quality studies.

Conclusion Good adherence is associated with a lower risk of severe asthma exacerbations. Future studies should use standardised methodology to assess adherence and inhaler technique.

\section{P153 DOES ASTHMA CONTROL, MOOD DISTURBANCE OR HEALTH STATUS INFLUENCE DAILY PHYSICAL ACTIVITY LEVELS IN PATIENTS WITH SEVERE ASTHMA?}

${ }^{1} \mathrm{~T}$ Pandya, ${ }^{2} \mathrm{~S}$ Majd, ${ }^{2} \mathrm{~S}$ Hewitt, ${ }^{2} \mathrm{~T}$ Harvey-Dunston, ${ }^{2} \mathrm{PH}$ Bradding, ${ }^{2} \mathrm{RH}$ Green, ${ }^{2} \mathrm{~S} J$ Singh, ${ }^{2}$ RA Evans. 'Manchester Medical School, Manchester, UK; ${ }^{2}$ Leicester NIHR Biomedical Research Unit, Glenfield Hospital, Leicester, UK

\subsection{6/thoraxjnl-2016-209333.296}

Introduction We aimed to assess the level of daily physical activity and investigate the relationship with asthma control, mood disturbance and health status in patients with severe asthma.

Methods Patients with severe asthma (step 4-5 of the British Thoracic Society guidelines), MRC dyspnoea grade $\geq 2$, were recruited from specialists in difficult-to-treat asthma. All patients were asked to wear SensewareTM activity monitor for seven days during waking hours with a minimum data requirement of eight hours per day for four days. ${ }^{1}$ Participants completed the Asthma Control Questionnaire (ACQ), Hospital Anxiety and Depression Scale (HADS), Chronic Respiratory Questionnaire (CRQ) and Asthma Quality of Life Questionnaire (AQLQ). Pearson's correlation coefficient was used to assess the relationship between physical activity, asthma control, mood disturbance and health status.

Results 45 participants (24 female, mean [SD] age 54 [13] yr, BMI 32 [7] kg/m ${ }^{2}$ ) provided written consent and physical activity data was available for 41 patients. The mean (SD) number of days of physical activity data available was 6.1 [1.9] days. The mean (SD) number of steps per day was 5258 [3030] with only 84 [82] active minutes. Only 16 patients achieved any moderate physical activity (3-4.5 metabolic equivalents [METS]) and for less than six minutes per day.

The mean [SD] ACQ was 13.6 [6.1], HADS anxiety and depression domains 6.5 [4.9] and 4.5 [2.8], respectively, CRQ Dyspnoea domain 3.4 [1.5], AQLQ environmental, symptoms, activity and emotional domains of 4.9 [1.4], 5.0 [1.4], 5.0 [1.2] and 4.9 [1.5], respectively. Table 1 shows the correlations between steps per day, and measures of asthma control, mood disturbance, and health status.

Abstract P153 Table 1 Relationship between steps per day and asthma control, mood disturbance and health status

\begin{tabular}{lll}
\hline Questionnaire & Correlation coefficient & $\boldsymbol{P}$ value \\
\hline ACQ & -0.16 & 0.28 \\
HADS - Anxiety & -0.17 & 0.28 \\
HADS - Depression & -0.13 & 0.41 \\
CRQ - Dyspnoea & 0.23 & 0.14 \\
AQLQ Activity & 0.34 & 0.02 \\
AQLQ Emotional & 0.17 & 0.26 \\
AQLQ Symptoms & 0.10 & 0.51 \\
AQLQ Environmental & 0.06 & 0.71 \\
AQLQ mean & 0.15 & 0.33 \\
\hline
\end{tabular}

Conclusions Patients with severe asthma demonstrated low levels of physical activity but there were no relationships with asthma control, mood disturbance or health status.

\section{REFERENCE}

1 Demeyers $\mathrm{H}$, et al. Chest 2014;146(2):318-27.

\section{P154 SAFETY OF TIOTROPIUM IN PRE-SCHOOL CHILDREN WITH SYMPTOMATIC PERSISTENT ASTHMA}

${ }^{1} \mathrm{H}$ Bisgaard, ${ }^{2} \mathrm{M}$ Vandewalker, ${ }^{3} \mathrm{~L}$ Graham, ${ }^{4} \mathrm{P}$ Moroni-Zentgraf, ${ }^{4} \mathrm{M}$ Engel, ${ }^{4} \mathrm{G}$ El Azzi, ${ }^{5} \mathrm{SD}$ Vulcu, ${ }^{6} \mathrm{H}$ Finnigan, ${ }^{7}$ EJLE Vrijlandt. ${ }^{1}$ University of Copenhagen, Department of Clinical Medicine, Gentofte Hospital, Copenhagen, Denmark; ${ }^{2}$ Clinical Research of the Ozarks, Inc., Columbia, MO, USA; ${ }^{3}$ Children's Healthcare of Atlanta, Atlanta, GA, USA; ${ }^{4}$ TA Respiratory Diseases, Boehringer Ingelheim Pharma GmbH and Co. KG, Ingelheim am Rhein, Germany; ${ }^{5}$ Translational Medicine and Clinical Pharmacology, Boehringer Ingelheim Pharma GmbH and Co. KG, Biberach an der Riss, Germany; ${ }^{6}$ Clinical Development and Medical Affairs/TA Respiratory Diseases, Boehringer Ingelheim Ltd, Bracknell, UK; ${ }^{7}$ Department of Paediatric Pulmonology and Paediatric Allergy, Beatrix Children's Hospital, University Medical Centre Groningen, University of Groningen, Groningen, The Netherlands

\subsection{6/thoraxjnl-2016-209333.297}

Introduction and objectives Asthma is the most common chronic disease of childhood (GINA 2015). For pre-school children whose asthma symptoms are not well controlled on inhaled corticosteroids, limited options are available for further treatment. Here, we evaluated the safety of once-daily (QD) tiotropium Respimat ${ }^{\circledR}$ (tioR) in patients aged 1-5 years with symptomatic persistent asthma.

Methods A Phase II/III, randomised, double-blind, placebo-controlled, parallel-group trial (NCT01634113) of tioR $5 \mu \mathrm{g}$, tioR $2.5 \mu \mathrm{g}$ or placebo Respimat ${ }^{\circledR}$ (pboR), administered QD in the afternoon for 12 weeks, each as add-on to usual maintenance therapy. Safety data, including post hoc analysis of a composite exacerbation end point derived from adverse events (AEs), are reported.

Results No AEs leading to treatment discontinuation or death were reported. The proportion of patients with any AEs was higher with pboR (73.5\%) than with tioR $5 \mu \mathrm{g}(58.1 \%)$ and 2.5

Abstract P154 Table 1 Composite asthma endpoint: Asthma exacerbation (broad) with pneumonia plus asthma worsening

\begin{tabular}{|c|c|c|c|}
\hline \multirow{5}{*}{$\begin{array}{l}\text { Composite end point } \\
\text { MedDRA preferred terms }\end{array}$} & \multicolumn{3}{|c|}{ Patients, n (\%) } \\
\hline & Tiotropium & Tiotropium & Placebo \\
\hline & Respimat $^{\oplus} 5 \mu \mathrm{g}$ & Respimat $^{\circledast 2} 2.5 \mu \mathrm{g}$ & Respimat $^{\oplus}$ \\
\hline & QD & QD & QD \\
\hline & $(n=31)$ & $(n=36)$ & $(n=34)$ \\
\hline Asthma exacerbation (broad)/ & $9(29.0)$ & $12(33.3)$ & $19(55.9)$ \\
\hline \multicolumn{4}{|l|}{ worsening + pneumonia } \\
\hline Asthma & $2(6.5)$ & $5(13.9)$ & $10(29.4)$ \\
\hline Bronchitis & $2(6.5)$ & $1(2.8)$ & $4(11.8)$ \\
\hline Bronchopneumonia & 0 & 0 & $1(2.9)$ \\
\hline Cough & $2(6.5)$ & $4(11.1)$ & $3(8.8)$ \\
\hline Dyspnoea & $1(3.2)$ & 0 & 0 \\
\hline Pneumonia & 0 & $1(2.8)$ & $2(5.9)$ \\
\hline Viral respiratory tract infection & $3(9.7)$ & $3(8.3)$ & $4(11.8)$ \\
\hline Wheezing & 0 & $2(5.6)$ & 0 \\
\hline
\end{tabular}

Treated set. Percentages calculated using total number of patients per treatment as denom inator. AE preferred terms defined by Medical Dictionary for Regulatory Activities version 17.1. 